\title{
Propagation of excitations induced by shear flow in nematic liquid crystals
}

\author{
J. T. Gleeson* \\ AT\&T Bell Laboratories, Murray Hill, New Jersey 07974 \\ and Physics Department, Kent State University, Kent, Ohio 44242 \\ P. Palffy-Muhoray \\ Liquid Crystal Institute, Kent State University, Kent, Ohio 44242
}

W. van Saarloos ${ }^{\dagger}$

AT\&T Bell Laboratories, Murray Hill, New Jersey 07974

(Received 12 October 1990)

\begin{abstract}
Under shear, nematic liquid crystals can exhibit localized excitations that propagate much faster than simple one-dimensional analysis can predict. We describe a two-dimensional theory in which the excitations are fronts between distinct solutions of the steady-state Ericksen-Leslie equations. We find that a front's speed is proportional to its width and also to the difference in an effective free energy that we also define. We consider the effects of shear in Hele-Shaw cells on the director field of a nematic liquid crystal, and find distinct classes of multiple steady-state director-field configurations. The front between two solutions always moves in the direction that decreases the total effective free energy. Although the speed cannot be calculated explicitly without knowing the details of the front region, it can be estimated selfconsistently using experimentally determined values for the observed front width. In this way we can obtain values for the front speed in reasonable agreement with observation.
\end{abstract}

\section{INTRODUCTION}

In viscous fingering experiments using nematic liquid crystals [1] in Hele-Shaw cells [2,3], as well as in the experiments of Zhu, Liu, and Bai [4,5], propagating excitations, or "halos," (cf. Fig. 1), have been observed that travel at very large speeds - typically $1 \mathrm{~cm} / \mathrm{s}$ but as high as $20 \mathrm{~cm} / \mathrm{s}$, which is much larger than the average flow speed $(\sim 0.1 \mathrm{~cm} / \mathrm{s})$. If one assumes that the director dis-

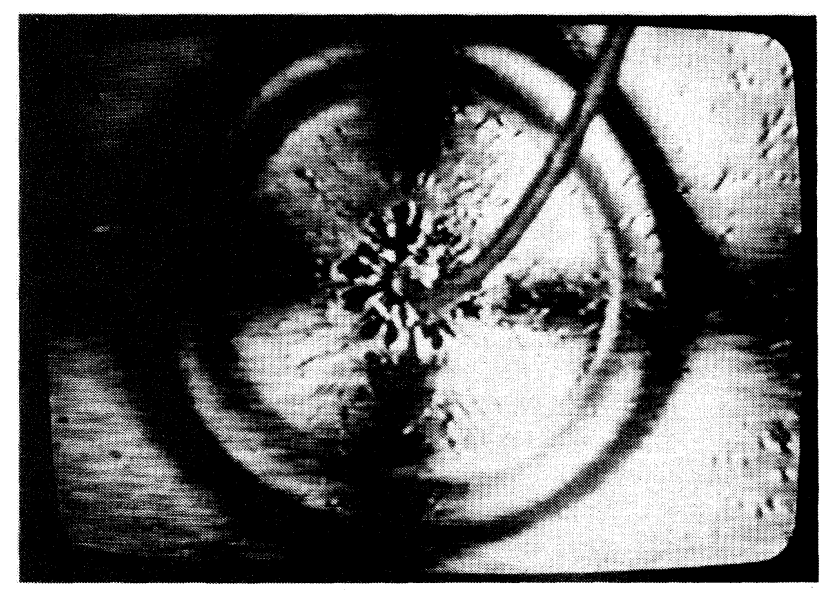

FIG. 1. Photograph of radial Hele-Shaw cell containing the liquid crystal $8 \mathrm{CB}$. It shows a diffuse halo that is approximately $0.4 \mathrm{~cm}$ wide and is propagating outward at $\sim 0.7 \mathrm{~cm} / \mathrm{s} . \eta$ is approximately 1500 . tortion occurs only over a length that scales with the plate separation $d$, dimensional analysis predicts a propagation speed

$$
c_{0} \equiv \frac{K}{\gamma_{1} d},
$$

where $K$ is an elastic constant and $\gamma_{1}$ is the viscosity for director rotation. Putting in values for a typical experiment, we obtain $c_{0} \approx 0.005 \mathrm{~cm} / \mathrm{s}$. Since $c_{0}$ is much less than the observed speeds, we must look further than this one-dimensional picture if we are to understand this phenomenon.

We describe an approach in which the director varies in two dimensions: perpendicular to the plates and along the direction of flow. Since in this case we must deal with lengths along both directions, and these may be quite different, dimensional analysis fails because it alone cannot tell us how to combine these two lengths. In our approach, the excitation is a front between two solutions of the steady-state Ericksen-Leslie [6] equations. The propagation speed is proportional to the width of the transition region between the two solutions and to the difference in an effective free energy that we also define. The Ericksen-Leslie equation for the director has a rich multiplicity of solutions because of both topological considerations and the inherent nonlinearity. We define for each solution an effective free energy by considering the work needed to rotate the director in the presence of viscous and elastic torques. We obtain the result that the front always moves in such a way as to reduce the total effective free energy. Since the width of the front and hence its speed cannot be calculated a priori, we use mea- 
surements of the front width and show reasonable agreement with observations of propagation speed.

\section{BACKGROUND}

Theoretical approaches [7-13] attempting to describe these phenomena have been in terms of the motion of smooth, nonlinear kinkline excitations (referred to as solitons) between two states of the director, $\widehat{\mathbf{n}}$. $[\widehat{\mathbf{n}}(\mathbf{r})$ is a unit vector field that describes the local direction of orientational order. See Ref. [1].] These calculations are onedimensional in that the structure of the director profile in the direction perpendicular to the plates is neglected. Furthermore, the state invaded by the kink is implicitly assumed to be linearly unstable. However, it is unrealistic to assume that the observed front is between a linearly stable state and an unstable state that is invaded by the stable state, because the unstable state would have to be prepared and then exist for at least the transit time of the front. This would be difficult to realize even were it the specific goal of the experimenter. Moreover, in a nematic liquid crystal, where director-orientation fluctuations are soft, it is probably not possible to prepare such a state.

Another important issue that has not been fully addressed previously is the effect of the director boundary condition at the glass plates. First, the director profile perpendicular to these planes cannot be homogeneous. Second, because of the boundary conditions, as well as the inherent nonlinearity of the problem, there is a large multiplicity of possible steady-state director profiles. These different director profiles cannot in general be continuously transformed into each other, i.e., they may be topologically inequivalent. We propose that the observed halo is a front propagating between different steady-state director profiles; we will show that there is a large number of solutions corresponding to different deformation modes. Three of these modes have been previously observed [14], and their linear stability has been calculated [15].

Given multiple solutions for the director profile and that the halos are fronts moving between them, what mechanism determines the propagation speed and the selection mechanism, i.e., which state does the system "prefer"? We are able to provide qualitative and intuitively appealing results by studying the Ericksen-Leslie [6] equations (which describe the coupling of the director to the flow field) in the approximation that the director remains in the shear plane and that the flow field is that of an isotropic fluid. We believe that this approach captures the essential physics responsible for the state selection and front speed problem. One attractive feature is that we can associate a "flow free energy," $\mathcal{F}_{\text {flow, }}$, with the viscous torque. The motion of fronts between different states is governed largely by the difference in the effective free energy: $\mathfrak{F} \equiv \mathscr{F}_{\text {el }}+\mathscr{F}_{\text {flow }}$ (where $\mathscr{F}_{\text {el }}$ is the Frank free energy, which is the energy cost of spatial variations in $\widehat{\mathbf{n}}$ ) between the states, so that the higher-energy state gives way to the lower. This picture allows us to solve the selection problem from an analysis of $\mathfrak{F}$ alone. We find that, for flow speeds comparable to those used in the experiments, states that are topologically different from the undistorted state become the lowest-effective-free-energy state.

In our approach, the direction of motion of a front between inequivalent states is determined by the difference in effective free energy of the two states, but the magnitude of the velocity depends also on the details of the director profile in the front region. However, if we assume the director distortions of the front extend over distances along the flow direction much larger than the plate separation, as appears to be the case in the experiment, we find that our analysis is consistent with front speeds that are much larger than $c_{0}$ or the fluid velocity, and of the same order as seen experimentally.

In order to understand the front dynamics completely, one must relax the assumption that the director remains in the shear plane; we expect some director profiles to escape. In this more general case, however, one cannot associate a unique flow energy with each state, since the work necessary to rotate the director from one state to another depends on the way in which the director distorts into the third dimension. We present arguments, however, that the picture developed here remains qualitatively the same even in this case. The full dynamical behavior of the fronts can be studied only with twodimensional numerical solutions of the Ericksen-Leslie equations, which are beyond the scope of this paper.

This paper proceeds as follows: in Sec. III we discuss the Ericksen-Leslie equations that describe this problem, as well as the simplifications we have made and their implications. Section III A presents our results for steadystate solutions of the director profile, including the multiplicities referred to above. In Sec. IV we discuss the relevant energies and their calculation. Section $V$ describes how the theory of front propagation applies to this problem and how one may calculate halo speed; Sec. VI gives a comparison between our results and experiments, and Sec. VII is our discussion and conclusions.

\section{THE ERICKSEN-LESLIE EQUATIONS}

Shear exerts viscous torque on the director of a nematic liquid crystal. The balance of this torque with that due to elasticity [1] and the corresponding backflow are described by the Ericksen-Leslie equations. Assuming that the director always lies in the plane of shear and that there is translational invariance perpendicular to this plane, $\hat{\mathbf{n}}$ is described uniquely by its angle $\theta(x, z, t)$ with the direction of the flow; the coordinates $x$ and $z$ are shown schematically in Fig. 2. In this geometry, $\cos \theta=\hat{\mathbf{n}} \cdot \widehat{\mathbf{x}}$ and the torque balance equation is

$$
\begin{aligned}
\gamma_{1}\left(\frac{\partial \theta}{\partial t}+u(z) \frac{\partial \theta}{\partial x}\right)= & K\left(\frac{\partial^{2} \theta}{\partial x^{2}}+\frac{\partial^{2} \theta}{\partial z^{2}}\right] \\
& +\frac{\partial u(z)}{\partial z}\left(\alpha_{2} \sin ^{2} \theta-\alpha_{3} \cos ^{2} \theta\right)
\end{aligned}
$$

where $u$ is the flow velocity in the $x$ direction, the $\alpha$ 's are Leslie viscosity coefficients, and $\gamma_{1}=\alpha_{3}-\alpha_{2} . K$ is an elastic constant (we have used the one-elastic-constant approximation and have verified that this does not affect the behavior significantly). Furthermore, in many ma- 


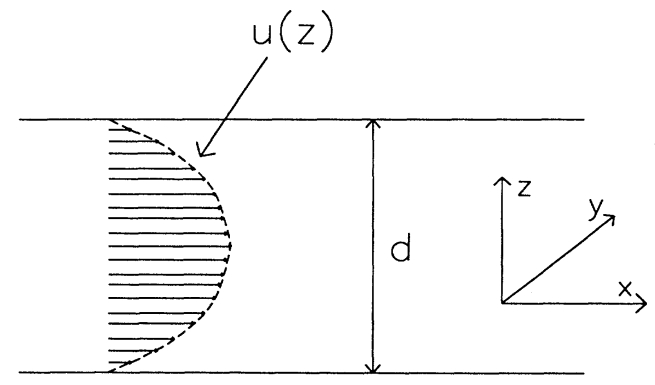

FIG. 2. Schematic of the geometry considered.

terials $\alpha_{2} \gg \alpha_{3}$, so we take $\alpha_{3}=0$. We have also verified that this affects the results very little. For simplicity we also neglect the effect of the director on $u$ and approximate it by the flow of an isotropic fluid determined solely by the externally imposed pressure gradient $\partial p / \partial x$. This, with no-slip boundary conditions gives

$$
u(z)=\frac{\partial p / \partial x}{\alpha^{\prime}}\left(\frac{d^{2}}{4}-z^{2}\right)=6 \frac{\bar{u}}{d^{2}}\left(\frac{d^{2}}{4}-z^{2}\right),
$$

where $\alpha^{\prime}$ is an average viscosity and $\bar{u}$ is the average flow speed. We have checked the validity of this assumption by calculating the true flow field $u(z)$ using the Ericksen-Leslie theory. The relative deviations from the assumed parabolic profile are less than $20 \%$, which is not significant at the level of this analysis.

\section{A. Steady-state solutions}

Using the above assumptions, Eq. (2) reduces to

$$
\frac{d^{2} \theta}{d z^{\prime 2}}+\eta z^{\prime} \sin ^{2}(\theta)=0
$$

where $z^{\prime} \equiv z / d$ and $\eta \equiv-12 \bar{u} d \alpha_{2} / K$ is the Ericksen number $\mathrm{Er}$. Er is a dimensionless number obtained by multiplying a characteristic shear rate (in our case $12 \bar{u} / d$ ) by the characteristic relaxation time for director distortions, $-\alpha_{2} d^{2} / K$. Typically, $\eta$ is of the order of 1000 [16]. Equation (4) is solved using fourth-order Runge-Kutta integration and the shooting method [17]; the bisection root-finding algorithm converges reliably to the correct shot. There is a large multiplicity of the solutions of this equation, largely unaddressed by other researchers, that plays a dominant role in determining the speed of the localized excitations referred to in the Introduction - this will be expanded on in Sec. IV. Boundary conditions consist of specifying the angle $\theta$ on the planes $z^{\prime}= \pm \frac{1}{2}$. The angle $\theta$ can be defined to within an additive integral multiple of $\pi$, giving rise to distinct classes of possible solutions. We assume strong anchoring for the homeotropic alignment used in our experiment, that is, the boundary conditions at the glass walls are

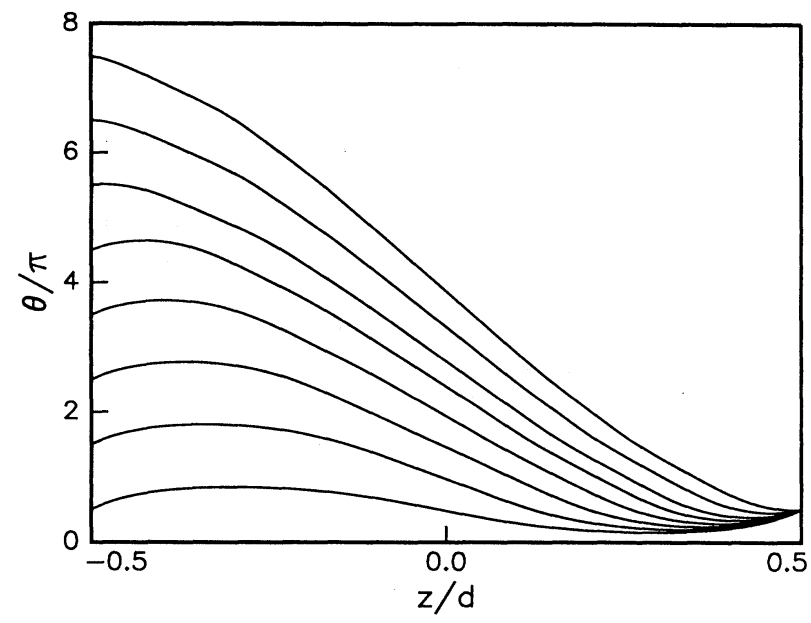

FIG. 3. Solutions of Eq. (4) for various winding numbers and $\eta=400$.

$$
\theta\left(z^{\prime}=\frac{1}{2}\right)=\frac{\pi}{2}, \quad \theta\left(z^{\prime}=-\frac{1}{2}\right)=\frac{\pi}{2}+m \pi .
$$

Traversing the cell from $z^{\prime}=-\frac{1}{2}$ to $z^{\prime}=\frac{1}{2}$, the director rotates through an angle $m \pi$, where $m$ is the "winding number" [18]. Classes of solutions are distinguished by their winding number. It is important to note that two solutions that differ in winding number by $\Delta m$ are topologically inequivalent and must be separated by a line defect (of strength $s=\Delta m / 2$ ). This immediately raises the question of which winding number (if any) is preferred. Figure 3 shows solutions of Eq. (4) for $\eta=400$ and various winding numbers.

In addition, we have found that for each winding number, a rich multiplicity of solutions exists. Unlike previous researchers, we have not assumed that

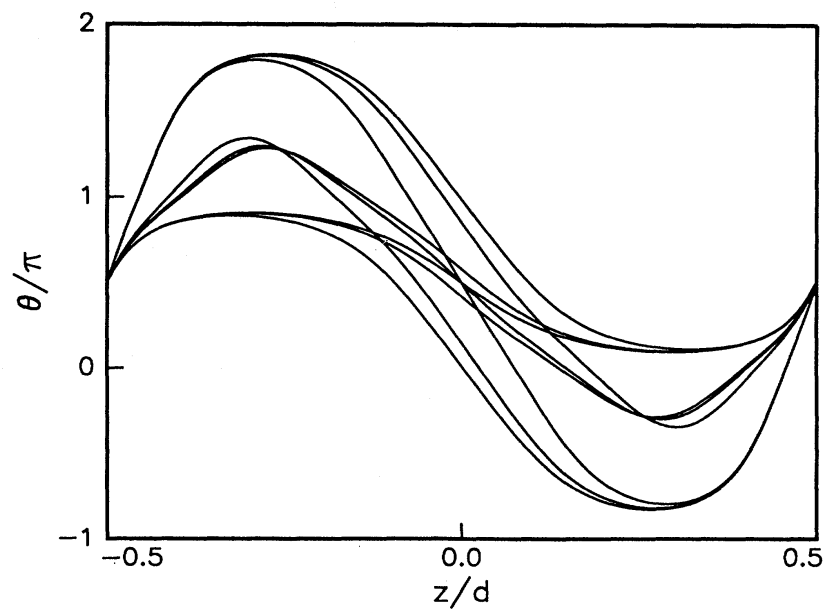

FIG. 4. Nine different solutions of Eq. (4) for $m=0$ and $\eta=900$. 


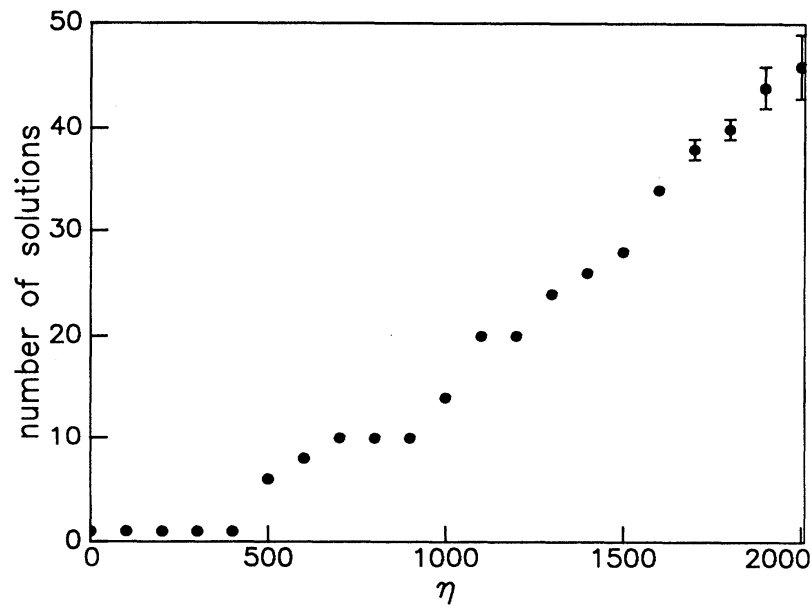

FIG. 5. The number of solutions of Eq. (4) increases dramatically as $\eta$ is increased. This is for winding number 0 . Essentially the same behavior is seen for other winding numbers. The error bars arise because at very large $\eta$, more solutions might be found by decreasing the mesh size used.

$\theta(z)-(m+1) \pi / 2$ is an odd function of $z$ [19]. These multiple solutions arise because Eq. (4) is nonlinear. Multiple solutions appear for $\eta>\eta_{c}(m)$, where $\eta_{c}(0) \approx 475$. Figure 4 shows nine different solutions of this equation for $\eta=900$ and $m=0$. The number of solutions, shown in Fig. 5, increases dramatically with $\eta$. Furthermore, this number increases in an interesting way. In Fig. 6 we plot $\theta\left(z^{\prime}=-\frac{1}{2}\right)-\pi / 2$, obtained from the shooting algorithm, versus $d \theta /\left.d z^{\prime}\right|_{z^{\prime}=1 / 2}$ when $\eta=1000$. As $\eta$ is in-

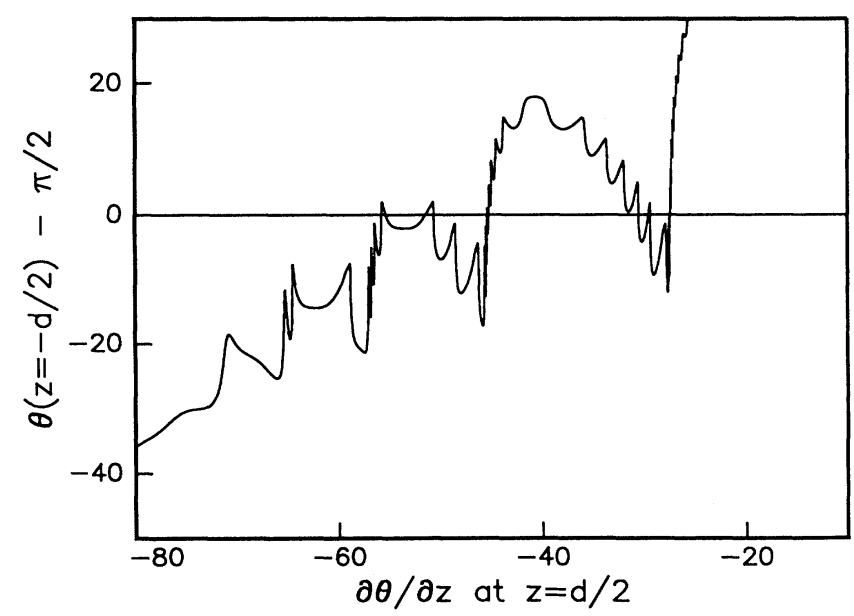

FIG. 6. $\theta\left(z^{\prime}=-\frac{1}{2}\right)-\pi / 2$ vs $\partial \theta /\left.\partial z^{\prime}\right|_{z^{\prime}=1 / 2}$ using the algorithm for Eq. (4); $\eta=1000$. Each zero of this function corresponds to a solution. As $\eta$ increases, the shape of the curve near the center becomes more intricate, leading to more solutions. creased, undulations of this curve increase in both frequency and magnitude, giving rise to many more solutions. The complexity of this curve illustrates the inherent richness of this problem.

Each solution represents a configuration with zero torque on the director everywhere and hence is a possible steady state.

\section{ENERGY CALCULATIONS}

Given a solution of Eq. (4), its Frank free energy per unit area $\mathcal{F}_{\text {el }}$ is [20]

$$
\mathcal{F}_{\mathrm{el}}=\frac{K}{2} \int_{-d / 2}^{d / 2}\left(\frac{\partial \theta}{\partial z}\right)^{2} d z
$$

The Frank energy is the free-energy cost of producing spatial variations in the director orientation. We define the flow energy $\mathfrak{F}_{\text {flow }}$

$$
\begin{aligned}
& \mathcal{F}_{\text {flow }}=-\int_{-d / 2}^{d / 2} \int_{\pi / 2}^{\theta(z)} \tau_{y} d \theta d z \\
&= \int_{-d / 2}^{d / 2} \int_{\pi / 2}^{\theta(z)} \frac{\partial u}{\partial z}\left(\alpha_{2} \sin ^{2} \theta-\alpha_{3} \cos ^{2} \theta\right) d \theta d z \\
&=\frac{1}{2} \int_{-d / 2}^{d / 2} \frac{\partial u}{\partial z}\left[\left(\alpha_{2}-\alpha_{3}\right) \theta(z)-\frac{1}{2}\left(\alpha_{2}+\alpha_{3}\right) \sin 2 \theta(z)\right. \\
&\quad+\text { const }] d z
\end{aligned}
$$

where $\tau$ is the director torque due to shear. The integration constant does not affect the calculation and will be ignored. We stress that this is not an energy in a thermodynamic sense, but is the integrated work done by the torque due to shear in rotating the director through $\theta(z)$. In the general case in which the director escapes out of the shear plane, this work depends on the "path" of the director rotation from the undistorted case, i.e., on the detailed behavior of this rotation. However, by restricting the director to the shear plane, the work necessary to rotate the director is, according to Eq. (7), completely

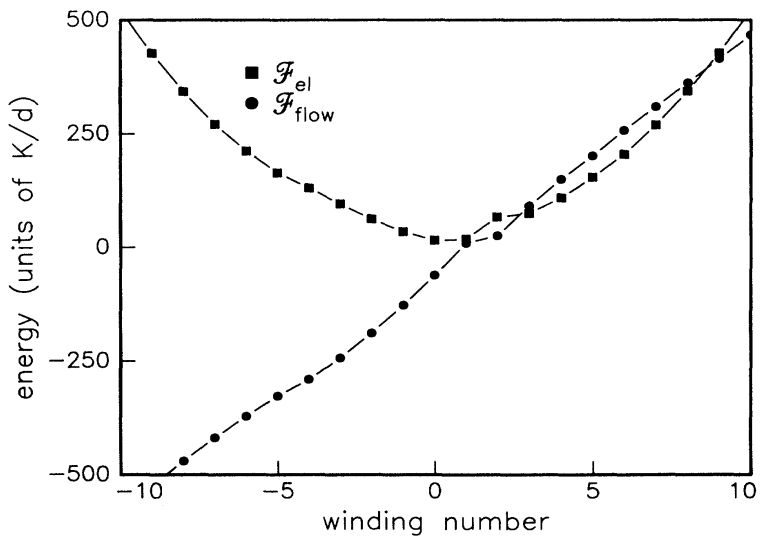

FIG. 7. Elastic energy and flow energy as a function of winding number for $\eta=400$. Note that the flow energy is nearly antisymmetric about $m=0$ while the Frank energy is nearly symmetric. 


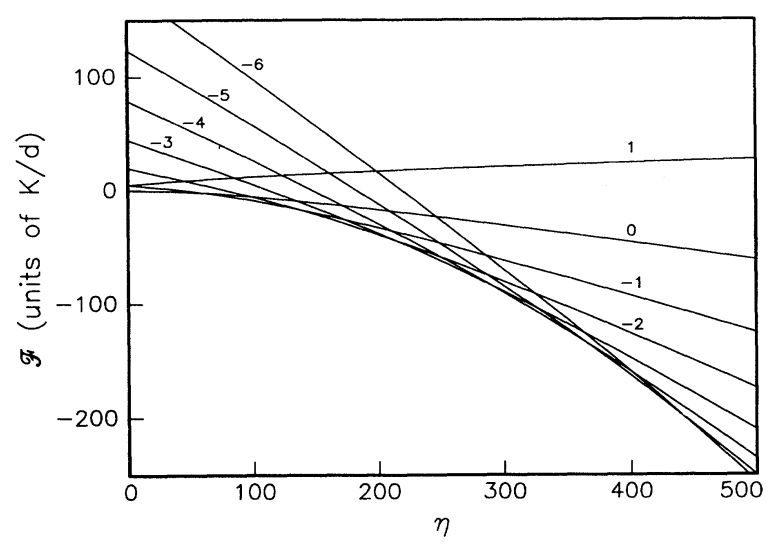

FIG. 8. Winding number of minimum energy solutions vs $\eta$.

specified by the angle $\theta(z)$; the restriction amounts to specifying the path of the rotation. We can think of $\mathscr{F}_{\text {flow }}$ as a free energy associated with the director torque caused by the shear. The sum of these two energy densities is the effective free energy $\mathcal{F}$. This energy is a Lyapunov functional from which Eq. (2) may be derived. $\mathcal{F}$ is also the total work needed to change the uniform state, $\theta \equiv \pi / 2$, to some solution of Eq. (4). We have computed both $\mathscr{F}_{\text {el }}$ and $\mathscr{F}_{\text {flow }}$ for solutions of Eq. (4). $\mathscr{F}_{\mathrm{el}}$ is roughly proportional to $\mathrm{m}^{2}$, while $\mathscr{F}_{\text {flow }}$ is roughly proportional to $m$. Solutions corresponding to $m>0$ and $m<0$ therefore have different effective free energy. This can be seen in Fig. 7 .

In Fig. 8, we show how the "most stable" solution (with the lowest $\mathcal{F}$ ) has smaller and smaller winding numbers as the flow is increased. Other authors [15] have found that solutions of Eq. (4) can be linearly unstable, which seems to be in qualitative agreement with our results.

\section{FRONT PROPAGATION}

We assume that there exists a propagating solution of Eq. (2) that connects two regions in which the director satisfies Eq. (4), such that in a reference frame moving at the speed of propagation the director field is time independent; in this comoving frame, Eq. (2) becomes

$$
\begin{aligned}
-\gamma_{1}(c-u) \frac{\partial \theta}{\partial x}= & K\left(\frac{\partial^{2} \theta}{\partial x^{2}}+\frac{\partial^{2} \theta}{\partial z^{2}}\right] \\
& +\frac{\partial u}{\partial z}\left(\alpha_{2} \sin ^{2} \theta-\alpha_{3} \cos ^{2} \theta\right),
\end{aligned}
$$

where $c$ is the frame speed (measured in the lab). Experimentally, the speed of the front in which we are interested is much larger than the flow velocity, and thus we neglect the second term in the above expression. Multiplying both sides by $\partial \theta / \partial x$ and integrating yields

$$
\gamma_{1} c \int_{-d / 2}^{d / 2} d z \int_{-x_{0}}^{x_{0}}\left(\frac{\partial \theta}{\partial x}\right)^{2} d x=\Delta \mathcal{F}
$$

(this result is derived in detail in Appendix A). We assume that far from the front $\left(|x| \gg>x_{0}\right), \partial \theta / \partial x \approx 0$. We note that Eq. (9) is only valid in the case where the director remains in the plane of shear. Nevertheless, as discussed in Appendix B, the results for the more general case are qualitatively the same. The right-hand side (rhs) of Eq. (9) is the total effective-free-energy density difference between the two states far from the right $\left(x=-x_{0}\right)$ and the left $\left(x=x_{0}\right)$ of the front region separating the two solutions. In the approximation that the director remains in the shear plane, this energy difference is the driving force for the front propagation.

Equation (9) is similar in form to the equation describing the motion of the interface between stable and metastable phases in a Ginzburg-Landau formalism [21,22]. The integral on the left-hand side (lhs) is always positive, thus the sign of $c$ is determined by the sign of $\Delta \mathscr{F}$. Thus, as in the case where the energy driving the front motion is a thermodynamic state variable, the front will always move in such a way that the more stable state invades the less stable, so that the total energy is always decreased.

An important feature of Eq. (9) or the GinzburgLandau formalism is that the front speed is proportional to the width of the front between two states. To understand this behavior for the fronts we are interested in, note that the lhs of Eq. (9) can be written as

$$
\begin{array}{rl}
\gamma_{1} c \int_{-d / 2}^{d / 2} d z \int_{-x_{0}}^{x_{0}}\left(\frac{\partial \theta}{\partial x}\right)^{2} & d x \\
& =\gamma_{1} c d \int_{-1 / 2}^{1 / 2} d z^{\prime} \int_{-x_{0}}^{x_{0}}\left[\frac{\partial \theta}{\partial x}\right]^{2} d x \\
& =\frac{\gamma_{1} c d}{l},
\end{array}
$$

which defines $l$. More specifically, $l$ is the spatial extent of the front in the $x$ direction, i.e., the length of the interval on $x$ where $\partial \theta / \partial x$ deviates appreciably from zero. This is approximately equal to the experimentally observed front width. For a front between two states with a difference in effective free energy $\Delta \mathscr{F}$, Eq. (9) becomes

$$
\gamma_{1} c \frac{d}{l}=\Delta \mathscr{F}
$$

and the speed is given by

$$
c=\frac{l \Delta \mathscr{F}}{\gamma_{1} d}=\frac{l \Delta \mathscr{F}}{K} c_{0},
$$

where $c_{0}=K / \gamma_{1} d$ is the characteristic speed for the material. This is our main result: the front's propagation speed is determined not only by its width but also by the energy difference between the two states that bracket it.

As seen in Fig. 8, the effective-free-energy difference between the most stable solution and the $m=0$ solution can be of order $10^{2} \mathrm{~K} / d$ or more. We argue below, however, that the transition between states with $\Delta m \gg 2$ is expected to split up into a series of $\Delta m=2$ transitions. For these, Fig. 8 shows that $\Delta \mathscr{F}$ is much smaller, of order $(10-30) K / d$. As noted earlier, for typical nematic liquid 
crystals, $c_{0} \approx 10^{-2} \mathrm{~cm} / \mathrm{s}$. Moreover, we observe that the spatial extent $l$ of a front is of the order of $0.1 \mathrm{~cm}$, so that $l / d \approx 20$. Thus with these estimates we see that Eq. (12) is consistent with front speeds up to several $\mathrm{cm} / \mathrm{s}$.

We stress that the estimate above shows only that it is natural to expect large front speeds. To predict the speed one must solve the front structure explicitly to compute the rhs of Eq. (9) (or its generalization to the case where the director escapes the shear plane). This would require a full two-dimensional numerical solution of Eq. (2) that is beyond the scope of this paper.

We can only make speculations about effects caused by the director escaping out of the plane of shear; other researchers have found that solutions of Eq. (4) can be linearly unstable to this [15]. A stationary disclination between two states with a difference in winding number of 2 corresponds to a defect of strength 1 . It is well known [23] that this will reduce its strain energy (and cease to be singular) by escaping into the third dimension (out of the shear plane in the geometry considered). We similarly expect a moving defect to distort out of the shear plane but do not expect this to change the qualitative picture. Quantitatively, it appears that the effect would be to shift the transitions to higher values of $\eta$, since the torque is decreased by $\cos ^{2} \phi$ (where $\phi$ is the director angle with the normal to the shear plane) when the director no longer remains in the shear plane. It is also well known that static disclinations with $s>1$ will break up into a number of weaker disclinations such that the sum of their strengths is the strength of the original defect [24], and there is no reason moving defects should not do the same. For example, if the director field is in an $m=3$ state and the flow is suddenly reversed so that the $m=-3$ state becomes stable, we expect that three $s=1$ defects will be generated; this is perhaps why in director waves, multiple fronts are often seen when the flow is reversed [4].

\section{COMPARISON WITH EXPERIMENTAL RESULTS}

In our experiments on radial Hele-Shaw cells filled with the liquid crystal octylcyanobiphenyl (8CB), we have observed halo speeds up to $\sim 0.7 \mathrm{~cm} / \mathrm{s}$ with an apparent front width of $\sim 0.4 \mathrm{~cm}$. The plate separation was 0.0025 $\mathrm{cm}$ and $\eta=1800$. This diffuse halo is likely a front between two topologically equivalent states. In this range of $\eta$, we expect a large number of solutions, so we cannot say specifically which ones are present. The order of magnitude of $\Delta \mathcal{F}$ between these types of states, however, is $\approx 10 \mathrm{~K} / d$. This our estimate for the halo speed is $\approx 1600 c_{0} \approx 0.8 \mathrm{~cm} / \mathrm{s}$. Other experiments on "director waves" in the nematic phase of $\mathrm{N}-(o-$ methoxybenzylidene)-p-butylaniline (MBBA) [4] showed speeds of up to $20 \mathrm{~cm} / \mathrm{s}$. In addition, the width of the front in the direction of motion can be inferred from the photographs to be $1.8 \mathrm{~mm}$ (the distance between planes was $0.050 \mathrm{~mm}$ ). These experiments were at much higher values of $\eta\left(\approx 1.5 \times 10^{4}\right)$. The effective-free-energy difference must be $\approx 10^{2} \mathrm{~K} / d$ in this case to arrive at the correct order of magnitude for the observed speed. This is not unreasonable at such large $\eta$.
It has also been observed by us as well as others [4] that the propagation of these excitations is most dramatic when the source of the flow is abruptly reversed. This also is in good qualitative agreement with our theory; it can be seen in Fig. 7 that when the sign of the driving flow is changed, there is an exchange of stability between classes with $m$ and $-m$.

\section{CONCLUSIONS}

In summary, we have presented a two-dimensional theory that predicts the existence of propagating fronts in a nematic liquid crystal subject to shear flow. These fronts are transition regions between steady-state solutions of the director-torque-balance equation. Using this theory, we have obtained an expression for the front velocity; it is proportional to the width of the front and the difference in the effective free energy of two states separated by the front. Calculating the width of the front is straightforward in principle, but requires the solution of a nonlinear partial differential equation (which may be singular), which is beyond the scope of this paper. The velocity dependence on front width is in reasonable agreement with experimental results. Our expression also exhibits semiquantitative agreement with experimental results on front speeds in two different systems.

\section{ACKNOWLEDGMENTS}

We thank P. E. Cladis and R. G. Larson for helpful discussions. J.T.G. acknowledges support from Natural Sciences and Engineering Research Council (Canada).

\section{APPENDIX A: DERIVATION OF EQ. (5)}

The first term on the rhs of Eq. (2) is

$$
K\left(\frac{\partial^{2} \theta}{\partial x^{2}}+\frac{\partial^{2} \theta}{\partial z^{2}}\right) \text {. }
$$

We multiply this by $\partial \theta / \partial x$ and integrate over the region depicted in Fig. 9:

$$
K \int_{-d / 2}^{d / 2} \int_{-x_{0}}^{x_{0}}\left(\frac{\partial^{2} \theta}{\partial x^{2}}+\frac{\partial^{2} \theta}{\partial z^{2}}\right) \frac{\partial \theta}{\partial x} d z d x
$$

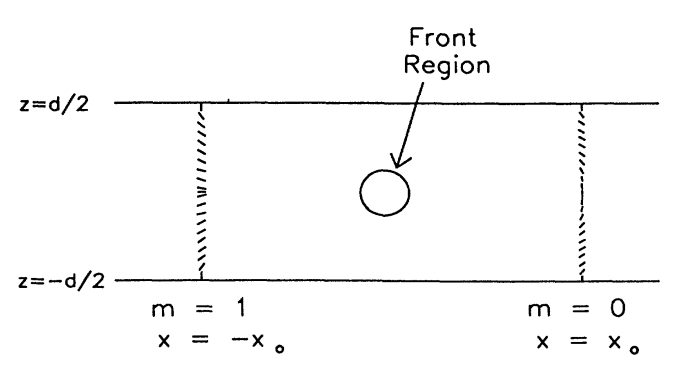

FIG. 9. Schematic of region of integration for Eq. (8). The distance $x_{0}$ of the boundaries from the front is much larger than l. 
where $x_{0}$ is some length larger than $l$ and we have placed the center of the front at $x=0$. The first term in the integrand can be rewritten as

$$
\frac{K}{2} \int_{-d / 2}^{d / 2} \int_{-x_{0}}^{x_{0}} \frac{\partial}{\partial x}\left(\frac{\partial \theta}{\partial x}\right)^{2} d z d x
$$

which can be integrated once; since by definition $\partial \theta / \partial x \equiv 0$ at $x= \pm x_{0}$, this term vanishes. The second term is rewritten as

$$
\frac{K}{2} \int_{-d / 2}^{d / 2} \int_{-x_{0}}^{x_{0}} \frac{\partial \theta}{\partial x} \frac{\partial}{\partial z} \frac{\partial \theta}{\partial z} d z d x
$$

and integrated by parts: $\partial \theta / \partial x$ vanishes on the lateral boundaries $(z= \pm d / 2)$ where strong anchoring is assumed; we thus have

$$
-K \int_{-d / 2}^{d / 2} \int_{-x_{0}}^{x_{0}} \frac{\partial \theta}{\partial z} \frac{\partial^{2} \theta}{\partial z \partial x} d z d x
$$

which can be rewritten as

$$
\frac{-K}{2} \int_{-d / 2}^{d / 2} \int_{-x_{0}}^{x_{0}} \frac{\partial}{\partial x}\left(\frac{\partial \theta}{\partial z}\right)^{2} d z d x
$$

or

$$
-\left(\left.\mathscr{F}_{\mathrm{el}}\right|_{x_{0}}-\left.\mathscr{F}_{\mathrm{el}}\right|_{-x_{0}}\right)=\Delta \mathscr{F}_{\mathrm{el}}
$$

using the definition of $\mathcal{F}_{\text {el }}$ of Eq. (6).

The second term on the rhs of Eq. (2), when multiplied by $\partial \theta / \partial x$ and integrated over the same region as we considered above, is

$$
\int_{-d / 2}^{d / 2} \int_{-x_{0}}^{x_{0}} \frac{\partial u}{\partial z}\left(\alpha_{2} \sin ^{2} \theta-\alpha_{3} \cos ^{2} \theta\right) \frac{\partial \theta}{\partial x} d z d x
$$

which we rewrite as

$$
\int_{-d / 2}^{d / 2} \int_{\theta\left(z,-x_{0}\right)}^{\theta\left(z, x_{0}\right)} \frac{\partial u}{\partial z}\left(\alpha_{2} \sin ^{2} \theta-\alpha_{3} \cos ^{2} \theta\right) d \theta d z
$$

which becomes

$$
\begin{aligned}
& \frac{1}{2} \int_{-d / 2}^{d / 2} \frac{\partial u}{\partial z} \gamma_{1}\left[\theta\left(z,-x_{0}\right)-\theta\left(z, x_{0}\right)\right] \\
& \quad-\left(\alpha_{2}+\alpha_{3}\right)\left[\sin 2 \theta\left(z,-x_{0}\right)-\sin 2 \theta\left(z, x_{0}\right)\right] d z .
\end{aligned}
$$

That is, recalling the definition in Eq. (7),

$$
-\left(\left.\mathscr{F}_{\text {flow }}\right|_{x_{0}}-\left.\mathscr{F}_{\text {flow }}\right|_{-x_{0}}\right)=\Delta \mathscr{F}_{\text {flow }}
$$

Combining the two terms gives Eq. (9).

\section{APPENDIX B: GENERALIZATION TO INCLUDE ESCAPE}

The results presented so far are for the specific case where the director always remains in the shear plane. Since this is almost certainly not the case experimentally, we present arguments here that the essential physics remains the same and that our method should still be valid in this more general case. When the director escapes out of the shear plane, forming an angle $\phi$ with this plane, Eq. (2) becomes

$$
\begin{aligned}
\gamma_{1} \cos \phi\left(\frac{\partial \theta}{\partial t}+u \frac{\partial \theta}{\partial x}\right)=K[ & \cos \phi\left[\frac{\partial^{2} \theta}{\partial x^{2}}+\frac{\partial^{2} \theta}{\partial z^{2}}\right] \\
& \left.-2 \sin \phi\left(\frac{\partial \theta}{\partial x} \frac{\partial \phi}{\partial x}+\frac{\partial \phi}{\partial z} \frac{\partial \theta}{\partial z}\right)\right] \\
& +\frac{\partial u}{\partial z} \cos \phi\left(\alpha_{2} \sin ^{2} \theta-\alpha_{3} \cos ^{2} \theta\right) .
\end{aligned}
$$

The torque balance equation that describes the behavior of the escape angle $\phi$ is

$$
\begin{aligned}
& \gamma_{1}\left(\frac{\partial \phi}{\partial t}+u \frac{\partial \theta}{\partial x}\right]= \frac{\partial u}{\partial z}\left(\alpha_{2}+\alpha_{3}\right) \cos \theta \sin \theta \cos \phi \sin \phi \\
&+K\left\{\frac{\partial^{2} \phi}{\partial x^{2}}+\frac{\partial^{2} \phi}{\partial z^{2}}\right. \\
&+\cos \phi \sin \phi\left[\left[\frac{\partial \theta}{\partial x}\right]^{2}\right. \\
&\left.\left.+\left(\frac{\partial \theta}{\partial z}\right]^{2}\right]\right\}
\end{aligned}
$$

We make the transformation to the comoving frame as before. The first equation is multiplied by $\cos \phi(\partial \theta / \partial x)$ and the second by $\partial \phi / \partial x$ and both are integrated over the same region as before. The procedure outlined in Appendix $A$ is carried out and the two resulting equations are added term by term. The result is

$$
\begin{aligned}
c \gamma_{1} \int_{-d / 2}^{d / 2} \int_{-x_{0}}^{x_{0}} \cos ^{2} \phi\left(\frac{\partial \theta}{\partial x}\right)^{2}+\left(\frac{\partial \phi}{\partial x}\right)^{2} d x d z \\
=\Delta \mathscr{F}_{\mathrm{el}}+\int_{-d / 2}^{d / 2} \int_{-x_{0}}^{x_{0}} \frac{\partial u}{\partial z}\left[\cos ^{2} \phi\left(\alpha_{2} \sin ^{2} \theta-\alpha_{3} \cos ^{2} \theta\right) \frac{\partial \theta}{\partial x}+\left(\alpha_{2}+\alpha_{3}\right) \sin \theta \cos \theta \cos \phi \sin \phi \frac{\partial \phi}{\partial x}\right] d x d z
\end{aligned}
$$

where $\mathcal{F}_{\text {el }}$ is now

$$
\mathscr{F}_{\mathrm{el}}=\frac{K}{2} \int_{-d / 2}^{d / 2} \cos ^{2} \phi\left(\frac{\partial \theta}{\partial z}\right)^{2}+\left(\frac{\partial \phi}{\partial z}\right)^{2} d z
$$


The last integral in Eq. (B3) is rewritten as

$$
\int_{-d / 2}^{d / 2} \frac{\partial u}{\partial z} d z \int_{-x_{0}}^{x_{0}}\left[\alpha_{2}\left[\cos ^{2} \phi \sin ^{2} \theta \frac{\partial \theta}{\partial x}-\frac{\sin 2 \theta}{4} \frac{\partial}{\partial x} \cos ^{2} \phi\right]-\alpha_{3}\left[\cos ^{2} \phi \cos ^{2} \theta \frac{\partial \theta}{\partial x}+\frac{\sin 2 \theta}{4} \frac{\partial}{\partial x} \cos ^{2} \phi\right] d x\right],
$$

which becomes, after some manipulation,

$$
\int_{-d / 2}^{d / 2} \frac{\partial u}{\partial z} d z \int_{-x_{0}}^{x_{0}} \frac{\left(\alpha_{2}-\alpha_{3}\right)}{2} \cos ^{2} \phi \frac{\partial \theta}{\partial x}-\frac{\left(\alpha_{2}+\alpha_{3}\right)}{4} \frac{\partial}{\partial x}\left(\sin 2 \theta \cos ^{2} \phi\right) d x .
$$

The second term can be integrated once, leaving

$$
-\Delta \int_{-d / 2}^{d / 2} \frac{\partial u}{\partial z} d z \frac{\left(\alpha_{2}+\alpha_{3}\right)}{4} \sin 2 \theta \cos ^{2} \phi+\int_{-d / 2}^{d / 2} \frac{\partial u}{\partial z} d z \int_{-x_{0}}^{x_{0}} \frac{\left(\alpha_{2}-\alpha_{3}\right)}{2} \cos ^{2} \phi \frac{\partial \theta}{\partial x} d x
$$

In Eq. (B3) the first term of the coefficient of $c$ on the lhs is changed (from the solvable case where there is no escape) only by a positive factor that is not greater than unity, and there is an additional positive term, so that the sign on the lhs is the same. The last two terms in the integrand of Eq. (B3) differ only from the flow energy of the no-escape case by a positive factor not greater than unity. However, detailed knowledge of the front is necessary to evaluate the last term. Nevertheless, we do not expect qualitatively different behavior from the case where the director stays in the shear plane.

${ }^{*}$ Present address: Department of Physics, Princeton University, Princeton, NJ 08544.

†Present address: Instituut Lorentz, University of Leiden, Nieuwsteeg 18, 2311 SB Leiden, The Netherlands.

[1] For a general treatment of liquid crystal physics, see P. G. deGennes, The Physics of Liquid Crystals (Clarendon, Oxford, 1974).

[2] A. Buka, J. Kertesz, and T. Vicsek, Nature 323, 424 (1986).

[3] A. H. Buka, P. Palffy-Muhoray, and Z. Racz, Phys. Rev. A 36, 3984 (1987).

[4] Ghozhen Zhu, Phys. Rev. Lett. 49, 1332 (1982).

[5] Guozhen Zhu, Xingzhou Liu, and Naibing Bai, Phys. Lett. A 117, 229 (1986).

[6] J. L. Ericksen, Archs. Ration. Mech. Analysis 4, 231 (1960); F. M. Leslie, Quart. J. Mech. Appl. Math. 19, 357 (1966).

[7] Lin Lei, Shu Changqing, Shen Juelian, P. M. Lam, and Huang Yun, Phys. Rev. Lett. 49, 1335 (1982).

[8] Z. C. Liang, R. F. Shao, C. Q. Shu, L. Y. Wang, and L. Lei, Mol. Cryst. Liq. Cryst. Lett. 3, 113 (1986).

[9] R. F. Shao, S. Zheng, Z. C. Liang, C. Q. Xhu, and L. Lei, Mol. Cryst. Liq. Cryst. 144, 345 (1987).

[10] C. Q. Shu and L. Lei, Mol. Cryst. Liq. Cryst. 131, 47 (1985).

[11] L. Lei, C.Q. Shu, and G. Xu, J. Stat. Phys. 39, 633 (1985).
[12] G. Xu, C. Q. Shu, and L. Lei, Phys. Rev. A 36, 277 (1987).

[13] Y. Guo and Z. C. Ou-Yang, Phys. Rev. A 40, 2810 (1989).

[14] K. Hiltrop and F. Fischer, Z. Naturforsch. 31, 800 (1976).

[15] I. Zúñiga and F. M Leslie, J. Non-Newtonian Fluid Mech. 33, 123 (1989).

[16] We arrive at this estimate using the material constants for MBBA, a fluid velocity $\sim 0.1 \mathrm{~mm} / \mathrm{s}$, and a plate separation of $\sim 0.025 \mathrm{~mm}$.

[17] W. H. Press, B. P. Flannery, S. A. Teukolsky, and W. T. Vetterling, Numerical Recipes (Cambridge University, Cambridge, 1986), p. 582.

[18] Note that this is not tumbling, in which there are no configurations with zero torque on the director and hence no steady-state solutions. During tumbling, the director winding up is a time-dependent solution.

[19] P. Palffy-Muhoray and J. T. Gleeson (unpublished).

[20] See Ref. [1] for a detailed discussion of nematic elasticity.

[21] P. E. Cladis, W. van Saarloos, D. Huse, J. S. Patel, J. W. Goodby, and P. L. Finn, Phys. Rev. Lett. 62, 1764 (1989).

[22] M. A. Anisomov, P. E. Cladis, E. E. Gorodetskii, D. A. Huse, V. E. Podneks, V. G. Taratuta, W. van Saarloos, and V. P. Voronov, Phys. Rev. A 41, 6749 (1990).

[23] P. E. Cladis and M. Kléman, J. Phys. 33, 591 (1972).

[24] The energy in the strain field associated with a defect of strength $s$ is proportional to $s^{2}$ and thus is decreased by the breakup described. 


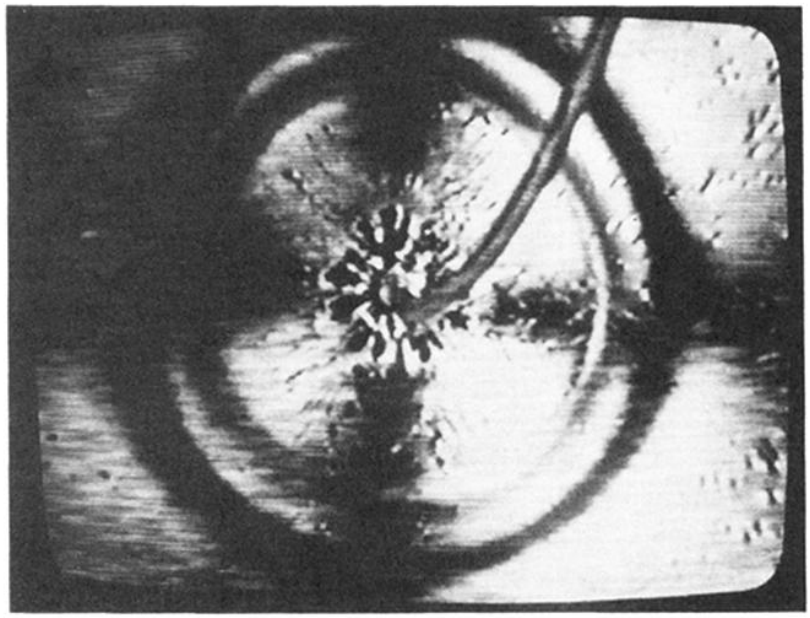

FIG. 1. Photograph of radial Hele-Shaw cell containing the liquid crystal $8 \mathrm{CB}$. It shows a diffuse halo that is approximately $0.4 \mathrm{~cm}$ wide and is propagating outward at $\sim 0.7 \mathrm{~cm} / \mathrm{s}$. $\eta$ is approximately 1500 . 